\title{
The CASCADE trial: effectiveness of ceramic versus PEEK cages for anterior cervical discectomy with interbody fusion; protocol of a blinded randomized controlled trial
}

Mark P Arts ${ }^{1 *}$, Jasper FC Wolfs ${ }^{1}$ and Terry P Corbin ${ }^{2}$

\begin{abstract}
Background: Anterior cervical discectomy with interbody fusion cages is considered the standard surgical procedure in patients with cervical disc herniation. However, PEEK or metal cages have some undesirable imaging characteristics, leading to a search for alternative materials not creating artifacts on images; silicon nitride ceramic. Whether patients treated with silicon nitride ceramic cages have similar functional outcome as patients treated with PEEK cages is not known. We present the design of the CASCADE trial on effectiveness of ceramic cages versus PEEK cages in patients with cervical disc herniation and/or osteophytes.

Methods/Design: Patients (age 18-75 years) with monoradicular symptoms in one or both arms lasting more than 8 weeks, due to disc herniation and/or osteophytes, are eligible for the trial. The study is designed as a randomized controlled equivalence trial in which patients are blinded to the type of cage for 1 year. The total follow-up period is 2 years. The primary outcome measure is improvement in the Neck and Disability Index (NDI). Secondary outcomes measures include improvement in arm pain and neck pain (VAS), SF-36 and patients' perceived recovery. The final elements of comparison are perioperative statistics including operating time, blood loss, length of hospital stay, and adverse events. Lateral plane films at each follow-up visit and CT scan (at 6 months) will be used to judge fusion and the incidence of subsidence. Based on a power of $90 \%$ and assuming $8 \%$ loss to follow-up, 100 patients will be randomized into the 2 groups. The first analysis will be conducted when all patients have 1 year of followup, and the groups will be followed for 1 additional year to judge stability of outcomes.
\end{abstract}

Discussion: While the new ceramic cage has received the CE Mark based on standard compliance and animal studies, a randomized comparative study with the golden standard product will provide more conclusive information for clinicians. Implementation of any new device should only be done after completion of randomized controlled effectiveness trials.

Keywords: Anterior cervical discectomy and fusion, Silicon nitride, Polyetheretherketone, PEEK, Interbody spacers, Randomized controlled trial, Herniated disc

\footnotetext{
*Correspondence: m.arts@mchaaglanden.nl

${ }^{1}$ Department of Neurosurgery, Medical Center Haaglanden, PO Box 432, 2501, CK The Hague, The Netherlands

Full list of author information is available at the end of the article
} 


\section{Background}

Since the introduction of anterior approach to the cervical spine by Cloward [1] and Smith [2], a dispute has arisen about the best surgical treatment. The purpose of all anterior cervical surgical procedures is removal of the intervertebral disc in order to decompress the nerve root and alleviate radicular pain and/or myelopathy. However, cervical instability and segmental collapse with recurrent radicular pain has been documented after anterior discectomy. For this reason, most surgeons perform anterior discectomy with interbody fusion (ACDF) although there is controversy about the benefits of adding interbody fusion to the cervical discectomy technique [3-9].

Anterior discectomy with fusion (ACDF) using bone graft in dowel or block form can have complications associated with graft collapse and can be associated with pain at the donor site if the graft is harvested from the iliac crest [10]. Interbody fusion using metal or plastic cages to contain and reinforce bone graft has been shown to have several advantages over bone blocks [11]: 1) using a cage allows the surgeon to fill the space without harvesting bone from the iliac crest in most cases; local bone or a bone graft substitute can be used instead; 2) the strength of the cage material ensures preservation of the disc height; bone blocks can crumble, decreasing the disc height with consequent neuroforaminal stenosis; and 3) using a cage saves time in the operating room and reduces blood loss. If there is no iliac crest bone graft harvest, there is less postoperative pain, as well.

Both plastic and metal cages have disadvantages as well. The principal plastic material, polyetheretherketone (PEEK), is hydrophobic and a mild chronic fibrous tissue reaction develops around the implant [12]. PEEK is invisible on imaging and therefore dislodgement and subsidence are difficult to determine. The two most commonly used metals, stainless steel and titanium distort magnetic resonance imaging (MRI) and CT scans, making determination of fusion and evaluation of degeneration difficult[13,14]. At present, ACDF with a PEEK cage is considered the golden standard for cervical disc herniation by many surgeons $[15,19]$.

Recently, ceramic materials have been evaluated as alternatives for interbody fusion devices. The cortical ring of the new device is manufactured by study sponsor Amedica Corporation (Salt Lake City, Utah) from its

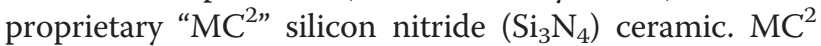
silicon nitride ceramic is a hydrophilic negative charged ceramic, which means that fluid (blood with nutrients) and proteins attach to the material, facilitating bone cell adherence and incorporation of the material in the surrounding bone. Cancellous Structured Ceramic (CSC) is a porous version of the same $\mathrm{MC}^{2}$ silicon nitride ceramic (Figure 1). Therefore, the entirety of this device is manufactured from identical material. The CSC material

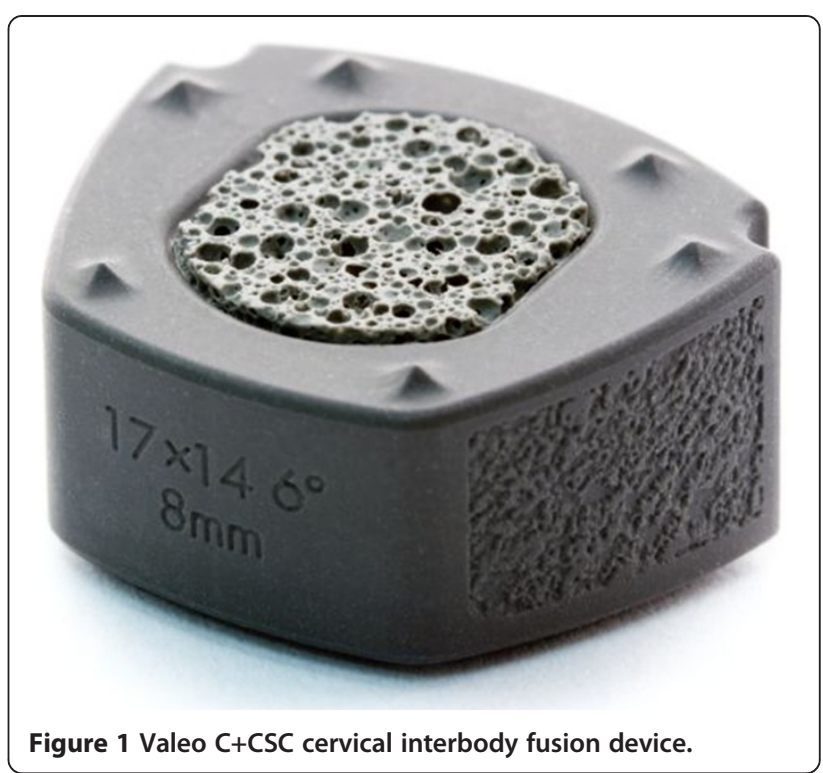

fills the center hole for the purpose of providing a scaffold for bone ingrowth. Because the surgical technique of this device does not involve harvesting autograft for packing into the pores of the porous trabecular structure, these implants afford the possibility of avoiding harvesting iliac crest autograft with attendant benefits of avoiding patient co-morbidities. Moreover, the silicon nitride material has desirable imaging properties. It is visible (like cortical bone) but does not create an artifact on CT or MR images (Figure 2).

The Valeo C+CSC cage has received the CE Mark based on preclinical testing and standards compliance, but has not been evaluated in a clinical study. Good clinical practice means that every new device should be compared to the golden standard prior to implementation of the device on a large scale. Therefore, it is necessary to perform a randomized controlled effectiveness trail on Valeo $\mathrm{C}+\mathrm{CSC}$ versus PEEK cages. In the CASCADE (CAncelous Structured Ceramic Arthrodesis DEvice) trial, we will randomly and blindly compare anterior discectomy with ceramic cages versus anterior discectomy with PEEK cages. In this equivalence trial, we hypothesize similar effectiveness and no difference in clinical improvement between the ceramic cage and the PEEK cage as measured with the Neck Disability Index (NDI). Moreover, radiological properties will be documented focusing on fusion and subsidence.

\section{Methods/design}

The study is designed as single center randomized controlled trial in which patients will be blinded for the allocated treatment. The follow-up period will be 2 years. All patients between 18 and 75 years old with monoradicular symptoms in one or both arms lasting 


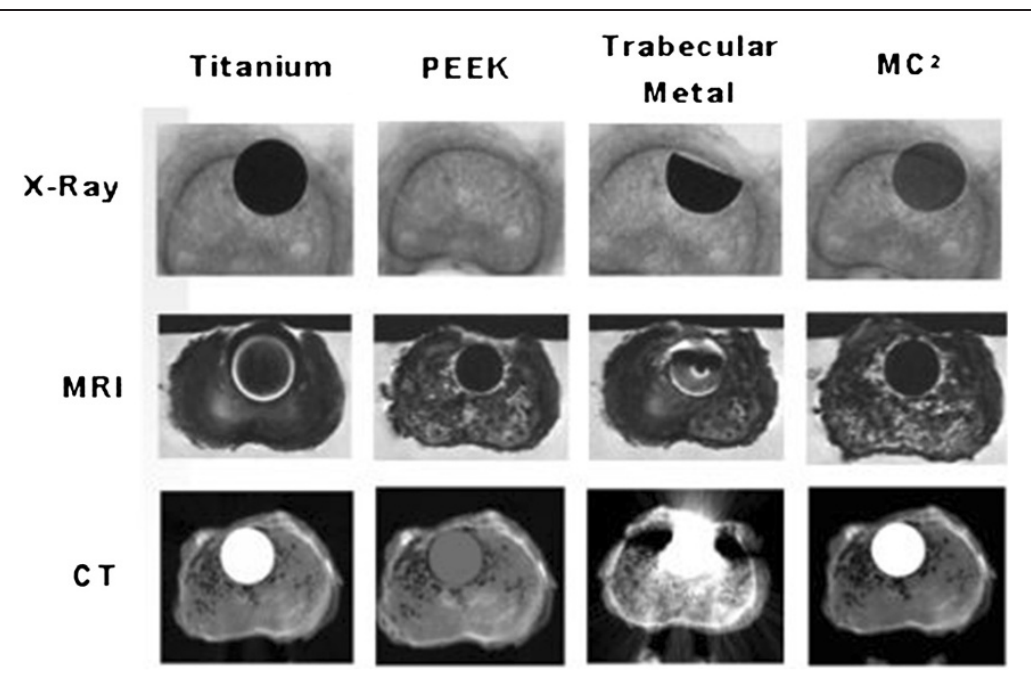

Figure 2 Silicon nitride, titanium, PEEK and trabecular metal imaging characteristics in a human cadaveric vertebra (unpublished data).

more than 8 weeks are eligible for the trial. MRI must confirm cervical disc herniation and/or osteophytes in accordance with clinical symptoms. Additional inclusion and exclusion criteria are listed in Table 1.

Patients are referred by a neurologist with MRI of the cervical spine. During the first visit to the neurosurgical outpatient clinic, the patient's history and a standard neurological examination will be documented. Based on our selection criteria, the neurosurgeon decides whether a patient is eligible for the trial.

\section{Informed consent and patients' safety}

The patient's written informed consent is obtained. The patient will be notified that they are free to withdraw their consent at any time. An independent physician has been appointed to answer patient questions and monitor the study, when necessary. Adverse events or complications will be monitored and followed up until stable or resolved. The project will be conducted according to the guidelines of Good Clinical Practice. The study has been reviewed and approved by the Medical Ethics Committee (METC) of Southwest Holland.

\section{Randomization procedure}

In order to eliminate confounding factors, the study is designed as a randomized controlled trial. Patients will be randomly allocated to the Valeo C+CSC or PEEK cages. Randomization will take place in the operating room within 6 weeks after inclusion. A randomization list was prepared by the data manager. A random numbergenerator was used to create the allocation sequence of blocks of 4, 6 , and 8 to ensure equal distribution of the randomized treatments. The data manager, who is not involved in the selection and allocation of patients, prepared numbered, coded, sealed envelopes containing the treatment allocation. In the operating room, after induction of anaesthesia, the surgeon will open the next numbered envelope and the allocated treatment will be performed. Patients will be kept blinded for the allocated treatment for 1 year.

\section{Surgical intervention}

All patients will be operated by the authors MA or JW, who both have extensive experience in cervical spine surgery and this ACDF technique. The patients will be positioned supine with their neck in neutral position or slightly extended under general anaesthesia. The affected cervical disc level will be verified with fluoroscopy. A small transverse incision will be made on the right side. Medial to the carotid sheath, the pre-vertebral space will be opened and the anterior cervical spine will be exposed. Caspar spreader and 2 distraction pins will be placed in the affected segment. A standard anterior discectomy with the aid of loupe magnification or microscope (depending on the surgeon's preference) will be performed in all cases. The posterior longitudinal ligament will be opened and the nerve root and dura will be decompressed adequately. Once the anterior discectomy has been performed, a PEEK interbody cage (Medicrea Manta, Lyon, France) (group 1) filled with local bone [20] obtained from removal of osteophytes, or the Valeo C+CSC spacer (Amedica Corporation, Salt Lake City, Utah) (group 2) will be placed within the intervertebral space under fluoroscopic guidance. The CSC core should be smeared with blood obtained by scratching the end plate after the disc space is prepared. No supplemental fixation (e.g., cervical plate) will be used in the procedure. If required, a vacuum drain will be placed and the wound will be closed in layers. 


\section{Table 1 Selection criteria for trial eligibility}

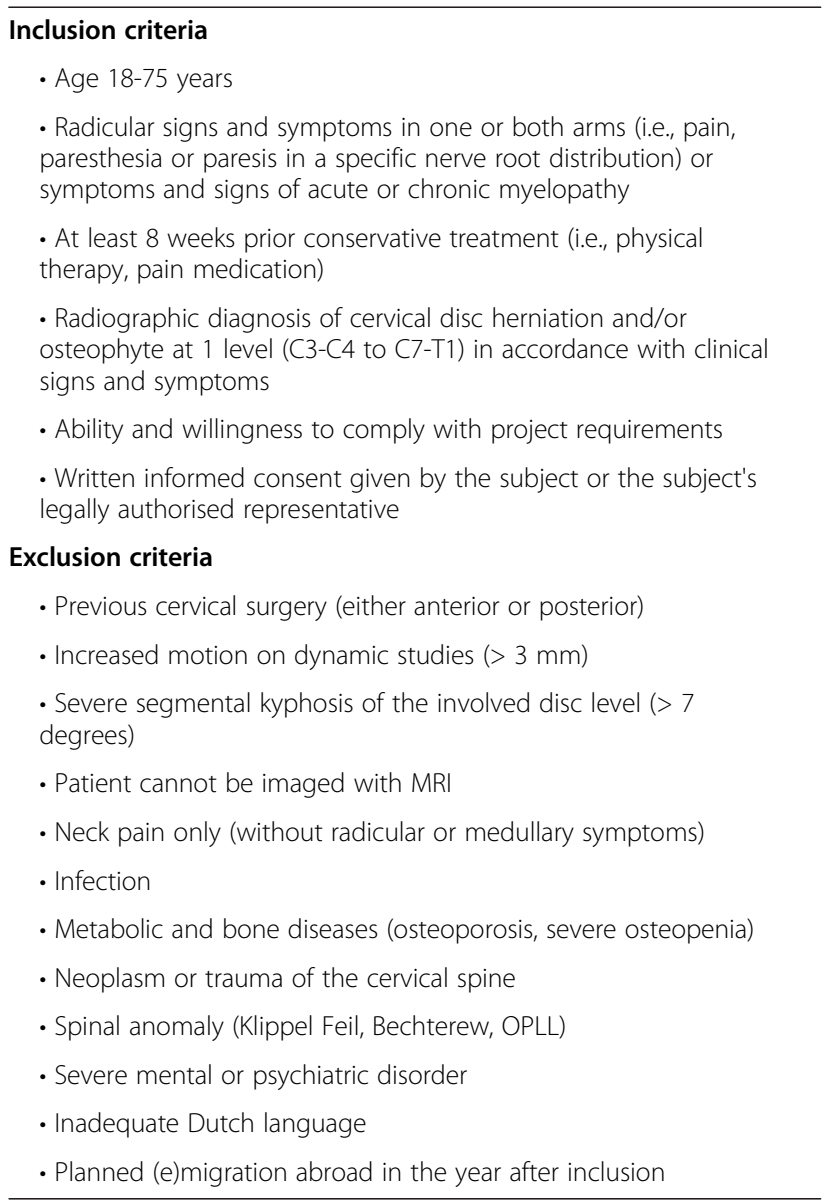

\section{Outcome assessment}

Baseline assessments include demographics, work status, smoking status, neck and arm pain, history of neck trauma, medical history, pain medication, body mass index, and neurological signs and symptoms. A general physical and neurological examination is performed before the patient is enrolled into the study and will be repeated at each subsequent visit.

We will assess the below described validated outcome parameters. Patients will not be informed about their previous scores. Follow-up examinations will take place by the surgeon at 3 months, 6 months, 12 months, and 24 months after randomization. The schedule of followup visits, radiographic studies and outcomes measures is included in Table 2. The following measures of outcomes will be used:

1) Neck Disability Index (primary outcome measure): The NDI is a patient-completed 10-item questionnaire on 3 different aspects; pain intensity, daily work related activities and non-work related activities. Each item is scored from 0 to 5 and the total score ranges from 0 (best score) to 50 (worst score). The NDI is a modification of the Oswestry Disability Index and has been shown to be reliable and valid for patients with cervical pathology [21,22] and has been validated in the Dutch language [23].

2) Short-Form 36 (SF-36): The SF-36 [24] is a generic health status questionnaire that can easily be filled out by the patient at home. The questionnaire consists of 36 items on physical and social status of the patient subdivided in 8 domains; 1 ) physical functioning, 2) physical restrictions, 3) emotional restrictions, 4) social functioning, 5) somatic pain, 6) general mental health, 7) vitality and 8) general health perception. The questions are scored on a scale of 0 (worst health) to 100 (ideal health). This questionnaire has been used frequently and is validated in surgical studies on spine pathology $[25,26]$.The Dutch language version has also been validated [27].

3) Pain intensity, measured by Visual Analogue Score (VAS) of arm and neck. The VAS of arm pain will measure the experienced pain intensity in the arm during the week before visiting the researcher. Pain will be assessed on a horizontal $100 \mathrm{~mm}$ scale varying from $0 \mathrm{~mm}$ (no pain) to $100 \mathrm{~mm}$ (worst pain imaginable). Patients do not see the results of earlier assessments and will score the pain experienced at the visit. Since many patients with radicular arm pain have neck pain as well, we will also measure the intensity of solitary neck pain. Reliability, validity and responsiveness of VAS have been shown [28].

4) Perceived recovery: Likert Scale is a 7-point scale measuring the perceived recovery, varying from 'complete recovery' to 'worse than ever'. This outcome scale has been used in previous studies and has been shown to be valid and responsive to change [29]. "Complete recovery" and "almost complete recovery" are defined as good result.

5) Radiological outcome: Anterior/posterior, and lateral views of the cervical spine will be performed at each visit. Displacement or subsidence of the device will be assessed by using the lateral radiograph. Only a change of $>3 \mathrm{~mm}$ will be considered clinically significant due to the margin of error in radiographic determination of displacement distances. Radiolucency at the cage-bone interface will be assessed as either present (if extending $50 \%$ of the length of the cage) or absent using a standard lateral radiograph. To verify fusion, CT axial images at $2 \mathrm{~mm}$ slices will be taken at the 6 months visit only.

6) Other outcome measures: In addition to the outcomes measures, data will be captured on details of the surgical procedure and hospitalization such as type and size of implant, duration of surgery, estimated blood loss, operative and postoperative complications, day of mobilization, and duration of 
Table 2 Data collection and outcome measures

\begin{tabular}{|c|c|c|c|c|c|c|}
\hline Measure & Pre-Op & Intra-Op & 3 months & 6 months & 12 months & 24 months \\
\hline Inclusion / exclusion criteria & $x$ & & & & & \\
\hline Product use, operative time, blood loss & & $x$ & & & & \\
\hline Neurological examination & $x$ & & $x$ & $x$ & $x$ & $x$ \\
\hline Neck Disability Index, Arm Pain VAS, Neck Pain VAS, SF-36, Recovery Likert & $x$ & & $x$ & $x$ & $x$ & $x$ \\
\hline Radiographs X-ray & $x$ & & $x$ & $x$ & $x$ & $x$ \\
\hline CT scan & & & & $x$ & & \\
\hline
\end{tabular}

hospitalization. Adverse events and reoperations will also be recorded by the operating surgeon.

The de-identified data from the initial visits, hospitalization and follow-up visits are entered by research nurses and physician assistants into a database via an Electronic Data Capture system (Acumen Healthcare Solutions, LLC, Plymouth, Minnesota, USA). Hospital staff schedules follow-up visits and supervises the collection of the patient-completed data forms. The source documents are kept filed in the hospitals where the procedures are performed.

Participating patients are given a small stipend to cover travel costs for follow-up visits. The cost of the procedure (including the interbody fusion devices) is covered by the patient's health insurance, but the expense of extra imaging and radiologist fees are paid by the study sponsor.

\section{Sample size and data analysis}

Sample size for this equivalence trial has been established using power analysis incorporating data from journal article reports of similar ACDF studies. For sample size calculations, we have used the change in NDI from pre-op to one year post-op cited in the literature for ACDF with carbon fiber reinforced PEEK cages: $10 \%$ reduction (improvement) with a standard deviation of $22 \%$ [30]. The Minimal Clinically Important Difference (MCID) for the NDI is 7.5 points or 15\% [31], which is the equivalence interval for sample size calculation. A 46-patient Valeo $\mathrm{C}$ enrollment has a power of .90 when compared with a PEEK study arm of the same size. Incorporating a one-year estimate of $8 \%$ loss to follow up, a total of 100 patients need to be enrolled.

The statistical significance of categorical data such as fusion will be tested using the Pearson Exact test. Parametric data will be evaluated using the Student's t-test. The first data analysis will be performed when all of the 1-year follow-up data is available for the primary endpoint. The two groups will be considered equivalent if the mean NDI improvement for the silicon nitride cage group is within a range from the mean of the PEEK group minus the NDI MCID to the PEEK mean plus the MCID. A repeated measurements analysis of variance for the primary outcome measure will also be performed in order to compare the evolving patterns over time.

The inclusion period started in December 2011 and 2 -year follow-up measures will be completed by the end of 2014 .

\section{Discussion}

A cervical radicular syndrome due to disc herniation is a well-known entity with an annual incidence rate of 83 per 100,000. Patients usually present with radicular arm pain and paraesthesiae, with or without neck pain. More than $90 \%$ of the patients have a favourable outcome with conservative treatment only [32] .Surgery is indicated whenever disabling pain persists. Anterior cervical discectomy $(A C D)$ is the basic surgical treatment of patients with radicular pain caused by cervical disc herniation. In 1958, Cloward first described anterior cervical decompression with the use of autologous iliac crest interbody graft (ACDF) to maintain disc height [1]. Smith and Robinson developed a technique using iliac crest bone blocks that was the standard for many year [2].There is still controversy about the benefits of adding interbody fusion to the cervical discectomy technique [4,10,33,34]. Frequently surgeons perform ACDF to maintain disc height and cervical alignment, and promote bony fusion to prevent instability [25].

Various prospective randomised trials have been performed comparing anterior discectomy with additional interbody fusion [3-9]. These results suggest that interbody fusion may not be necessary in all cases. However, definite conclusions could not be drawn due to methodological flaws such as small sample size, nonhomogenous patient population, undefined randomisation procedures and inconsistent outcome measures. Two large randomized trials are currently addressing the question of the value of adding fusion or cervical total disc replacements to the cervical discectomy procedure $[35,36]$. It is anticipated that ACDF will still be a viable choice for many patients even after these trials are completed.

Although there is no consensus on which interbody device to use, currently PEEK cages are considered as the golden standard for anterior cervical discectomy with fusion by many surgeons [15-19]. However, PEEK 
and titanium have some undesirable imaging characteristics that result in a demand for better cages with similar effectiveness. Figure 2 illustrates the superior imaging properties of $\mathrm{MC}^{2}$ ceramic material compared to PEEK, titanium and trabecular metal. Moreover, the CSC material fills the center hole for the purpose of providing a scaffold for bone ingrowth resulting in solid fusion and no subsidence of the cage. The CSC form of the material has performed well in an animal model [37] but the results and effectiveness need to be validated in humans. It is possible that solid fusion without signs of subsidence may lead to improved clinical outcome.

The present protocol of the CASCADE trial is designed to demonstrate the effectiveness and security of cancellous structured ceramic cages compared to the golden standard PEEK cages in patients treated with anterior cervical discectomy and fusion. Worldwide implementation of new devices should only be done after completion of randomized controlled trials. In our opinion, Level 1 evidence that government and health insurance organizations are demanding would not be possible without close cooperation between industry and researchers, but transparency is warranted and relevant conflicts of interest must be disclosed.

\section{Abbreviations}

CASCADE: Cancellous structured ceramic arthrodesis device; ACDF: Anterior cervical discectomy with fusion; ACD: Anterior cervical discectomy; PEEK: Polyetheretherketone; CSC: Cancellous structured ceramic; MRI: Magnetic resonance imaging; CT: Computer tomography; NDI: Neck disability index; VAS: Visual analogue scale.

\section{Competing interests}

Dr. Arts. Dr. Wolfs and Mr. Corbin are receiving financial support for their work on this study from Amedica Corporation.

\section{Authors' contributions}

MA designed the protocol, contributed to acquisition of data, is primary investigator and coordinator of the trial. JW has contributed to acquisition of data. TC has contributed to the case record forms, is responsible for the sample size calculation and analysis of the trial. All authors read and approved the final manuscript

\section{Acknowledgements}

The CASCADE trial is funded by Amedica Corporation. Furthermore we want to thank Ditte Varkevisser and Robin van Zijl for their work in making this trial possible.

\section{Author details}

'Department of Neurosurgery, Medical Center Haaglanden, PO Box 432, 2501, CK The Hague, The Netherlands. ${ }^{2}$ Corbin \& Company, Maple Grove, Minnesota, USA.

Received: 20 March 2013 Accepted: 13 August 2013

Published: 16 August 2013

\section{References}

1. Cloward RB: The anterior approach for removal of ruptured cervical disks. J Neurosurg 1958, 15:602-617.

2. Smith GW, Robinson RA: The treatment of certain cervical-spine disorders by anterior removal of the intervertebral disc and interbody fusion. J Bone Joint Surg Am 1958, 40-A:607-624.
3. Abd-Alrahman N, Dokmak AS, bou-Madawi A: Anterior cervical discectomy (ACD) versus anterior cervical fusion (ACF), clinical and radiological outcome study. Acta Neurochir (Wien) 1999, 141:1089-1092.

4. Barlocher CB, Barth A, Krauss JK, Binggeli R, Seiler RW: Comparative evaluation of microdiscectomy only, autograft fusion, polymethylmethacrylate interposition, and threaded titanium cage fusion for treatment of single-level cervical disc disease: a prospective randomized study in 125 patients. Neurosurg Focus 2002, 12:E4.

5. Dowd GC, Wirth FP: Anterior cervical discectomy: is fusion necessary? J Neurosurg 1999, 90:8-12.

6. Martins AN: Anterior cervical discectomy with and without interbody bone graft. J Neurosurg 1976, 44:290-295.

7. Rosenorn J, Hansen EB, Rosenorn MA: Anterior cervical discectomy with and without fusion. A prospective study. J Neurosurg 1983, 59:252-255.

8. Savolainen S, Rinne J, Hernesniemi J: A prospective randomized study of anterior single-level cervical disc operations with long-term follow-up: surgical fusion is unnecessary. Neurosurgery 1998, 43:51-55.

9. van den Bent MJ, Oosting J, Wouda EJ, van Acker RE, Ansink BJ, Braakman R: Anterior cervical discectomy with or without fusion with acrylate. A randomized trial. Spine (Phila Pa 1976) 1996, 21:834-839.

10. Jacobs WC, Anderson PG, Limbeek J, Willems PC, Pavlov P: Single or double-level anterior interbody fusion techniques for cervical degenerative disc disease. Cochrane Database Syst Rev 2004, 18(4):CD004958.

11. Ryken TC, Heary RF, Matz PG, Anderson PA, Groff MW, Holly LT, et al: Techniques for cervical interbody grafting. J Neurosurg Spine 2009, 11:203-220

12. Toth $J M$, Wang M, Estes BT, Scifert JL, Seim HB III, Turner AS: Polyetheretherketone as a biomaterial for spinal applications. Biomaterials 2006, 27:324-334.

13. Rudisch A, Kremser C, Peer S, Kathrein A, Judmaier W, Daniaux H: Metallic artifacts in magnetic resonance imaging of patients with spinal fusion. A comparison of implant materials and imaging sequences. Spine (Phila Pa 1976) 1998, 23:692-699.

14. Wang JC, Yu WD, Sandhu HS, Tam V, Delamarter RB: A comparison of magnetic resonance and computed tomographic image quality after the implantation of tantalum and titanium spinal instrumentation. Spine (Phila Pa 1976) 1998, 23:1684-1688.

15. Celik SE, Kara A, Celik S: A comparison of changes over time in cervical foraminal height after tricortical iliac graft or polyetheretherketone cage placement following anterior discectomy. J Neurosurg Spine 2007, 6:10-16.

16. Cho DY, Liau WR, Lee WY, Liu JT, Chiu CL, Sheu PC: Preliminary experience using a polyetheretherketone (PEEK) cage in the treatment of cervical disc disease. Neurosurgery 2002, 51:1343-1349.

17. Kulkarni AG, Hee HT, Wong HK: Solis cage (PEEK) for anterior cervical fusion: preliminary radiological results with emphasis on fusion and subsidence. Spine J 2007, 7:205-209.

18. Lied B, Roenning PA, Sundseth J, Helseth E: Anterior cervical discectomy with fusion in patients with cervical disc degeneration: a prospective outcome study of 258 patients (181 fused with autologous bone graft and 77 fused with a PEEK cage). BMC Surg 2010, 10:10.

19. Park HW, Lee JK, Moon SJ, Seo SK, Lee JH, Kim SH: The efficacy of the synthetic interbody cage and Grafton for anterior cervical fusion. Spine (Phila Pa 1976) 2009, 34:E591-E595.

20. Caroli E, Orlando ER, D'Andrea G, Ferrante L: Anterior cervical fusion with interbody titanium cage containing surgical bone site graft: our institution's experience in 103 consecutive cases of degenerative spondylosis. J Spinal Disord Tech 2007, 20:216-220.

21. Pietrobon R, Coeytaux RR, Carey TS, Richardson WJ, DeVellis RF: Standard scales for measurement of functional outcome for cervical pain or dysfunction: a systematic review. Spine (Phila Pa 1976) 2002, 27:515-522.

22. Vernon H, Mior S: The Neck Disability Index: a study of reliability and validity. J Manipulative Physiol Ther 1991, 14:409-415.

23. Vos CJ, Verhagen AP, Koes BW: Reliability and responsiveness of the Dutch version of the Neck Disability Index in patients with acute neck pain in general practice. Eur Spine J 2006, 15:1729-1736.

24. Ware JE Jr, Sherbourne CD: The MOS 36-item short-form health survey (SF-36). I. Conceptual framework and item selection. Med Care 1992, 30:473-483

25. Brazier JE, Harper R, Jones NM, O'Cathain A, Thomas KJ, Usherwood T, et al: Validating the SF-36 health survey questionnaire: new outcome measure for primary care. BMJ 1992, 305:160-164. 
26. Stansfeld SA, Roberts R, Foot SP: Assessing the validity of the SF-36 General Health Survey. Qual Life Res 1997, 6:217-224.

27. Van der Zee KSR: De psychometrische kwaliteiten van de MOS 36-item Short Form Health Survey (SF-36) in een Nederlandse populatie. T Soc Gezondheidsz 1993, 71:183-191.

28. Carlsson AM: Assessment of chronic pain. I. Aspects of the reliability and validity of the visual analogue scale. Pain 1983, 16:87-101.

29. Bombardier C: Outcome assessments in the evaluation of treatment of spinal disorders: summary and general recommendations. Spine (Phila Pa 1976) 2000, 25:3100-3103.

30. Peolsson A, Vavruch L, Oberg B: Disability after anterior decompression and fusion for cervical disc disease. Adv Physiol Educ 2002, 4:111-124.

31. Carreon LY, Glassman SD, Campbell MJ, Anderson PA: Neck Disability Index, short form-36 physical component summary, and pain scales for neck and arm pain: the minimum clinically important difference and substantial clinical benefit after cervical spine fusion. Spine J 2010 10:469-474.

32. Radhakrishnan K, Litchy WJ, O'Fallon WM, Kurland LT: Epidemiology of cervical radiculopathy. A population-based study from Rochester, Minnesota, 1976 through 1990. Brain 1994, 117(Pt 2):325-335.

33. Hirsch C: Cervical disc rupture: diagnosis and therapy. Acta Orthop Scand 1960, 30:186.

34. Nandoe Tewarie RD, Bartels RH, Peul WC: Long-term outcome after anterior cervical discectomy without fusion. Eur Spine J 2007, 16:1411-1416.

35. Arts MP, Brand R, van den Akker E, Koes BW, Peul WC: The NEtherlands Cervical Kinematics (NECK) Trial. Cost-effectiveness of anterior cervical discectomy with or without interbody fusion and arthroplasty in the treatment of cervical disc herniation; a double-blind randomised multicenter study. BMC Musculoskelet Disord 2010, 11:122.

36. Bartels RH, Donk R, van der Wilt GJ, Grotenhuis JA, Venderink D: Design of the PROCON trial: a prospective, randomized multi-center study comparing cervical anterior discectomy without fusion, with fusion or with arthroplasty. BMC Musculoskelet Disord 2006, 7:85.

37. Anderson $M C$, Olsen $R$ : Bone ingrowth into porous silicon nitride. J Biomed Mater Res A 2010, 92:1598-1605.

doi:10.1186/1471-2474-14-244

Cite this article as: Arts et al:: The CASCADE trial: effectiveness of ceramic versus PEEK cages for anterior cervical discectomy with interbody fusion; protocol of a blinded randomized controlled trial. $B M C$ Musculoskeletal Disorders 2013 14:244.

\section{Submit your next manuscript to BioMed Central and take full advantage of:}

- Convenient online submission

- Thorough peer review

- No space constraints or color figure charges

- Immediate publication on acceptance

- Inclusion in PubMed, CAS, Scopus and Google Scholar

- Research which is freely available for redistribution

Submit your manuscript at www.biomedcentral.com/submit
Biomed Central 